\title{
Dynamic generation of capillary waves
}

\author{
Hector D. Ceniceros and Thomas Y. Hou \\ Applied Mathematics, California Institute of Technology, Pasadena, California 91125
}

(Received 28 April 1998; accepted 27 January 1999)

\begin{abstract}
We investigate the dynamic generation of capillary waves in two-dimensional, inviscid, and irrotational water waves with surface tension. It is well known that short capillary waves appear in the forward front of steep water waves. Although various experimental and analytical studies have contributed to the understanding of this physical phenomenon, the precise mechanism that generates the dynamic formation of capillary waves is still not well understood. Using a numerically stable and spectrally accurate boundary integral method, we perform a systematic study of the time evolution of breaking waves in the presence of surface tension. We find that the capillary waves originate near the crest in a neighborhood, where both the curvature and its derivative are maximum. For fixed but small surface tension, the maximum of curvature increases in time and the interface develops an oscillatory train of capillary waves in the forward front of the crest. Our numerical experiments also show that, as time increases, the interface tends to a possible formation of trapped bubbles through self-intersection. On the other hand, for a fixed time, as the surface tension coefficient $\tau$ is reduced, both the capillary wavelength and its amplitude decrease nonlinearly. The interface solutions approach the $\tau=0$ profile. At the onset of the capillaries, the derivative of the convection is comparable to that of the gravity term in the dynamic boundary condition and the surface tension becomes appreciable with respect to these two terms. We find that, based on the $\tau=0$ wave, it is possible to estimate a threshold value $\tau_{0}$ such that if $\tau \leqslant \tau_{0}$ then no capillary waves arise. On the other hand, for $\tau$ sufficiently large, breaking is inhibited and pure capillary motion is observed. The limiting behavior is very similar to that in the classical KdV equation. We also investigate the effect of viscosity on the generation of capillary waves. We find that the capillary waves still persist as long as the viscosity is not significantly greater than surface tension. (c) 1999 American Institute of Physics. [S1070-6631(99)03005-6]
\end{abstract}

\section{INTRODUCTION}

Nonlinear water wave motion is a very interesting example of complex dynamics. An important event is the generation of capillary waves on the forward wave front. ${ }^{1-3}$ These capillary waves typically appear near the crest of the main wave where the local curvature is very large and the effect of surface tension becomes important. The understanding of these short waves is important in the remote sensing of sea surface because the fine structure associated with short wavelengths scatters electromagnetic radiation. Capillary waves are also believed to be a mechanism for extracting energy from wind-generated waves and may be significant in wave breaking. ${ }^{1,2}$

The phenomenon of capillary waves generated by steady steep gravity waves has been studied systematically by Longuet-Higgins in a series of papers, ${ }^{4-6}$ and by Crapper. ${ }^{7}$ Longuet-Higgins interprets the surface tension effect as a very unevenly concentrated pressure distribution on the surface, which combined with the effect of gravity, makes the capillary ripples appear near the crest of a steep wave. Using a perturbation technique, Longuet-Higgins obtains theoretical predictions for the capillary wavelength and amplitude in the approximation of steady Stokes waves. Longuet-Higgins and Dommermuth ${ }^{8}$ have also considered the time development of capillary waves, starting with the almost highest Stokes wave as initial data. A different motion, gravity- capillary waves of permanent form, has been investigated by Chen and Saffman ${ }^{9,10}$ and by Schwartz and VandenBroeck. ${ }^{11}$ They found that, in the maximum amplitude limit, the waves can self-intersect, forming trapped bubbles.

The effects of surface tension on breaking waves have been addressed by Tulin, ${ }^{12}$ who used a boundary element method developed by Wang, Yao, and Tulin. ${ }^{13}$ The remarkable simulations presented by Tulin show clearly the appearance of a capillary jump, as defined by Longuet-Higgins, ${ }^{14}$ near the wave crest. Here, as in Tulin's simulations, we deal with surface tension effects on unsteady breaking waves on the surface of a potential fluid but focus on periodic data only. By using a stable and spectrally accurate numerical method, we are able to compute not only up to the appearance of the capillary jump but also to follow the subsequent development of a very large curvature at and near the toe (the point where the capillaries seem to originate). The high accuracy of our method allows us to also capture in detail the small-scale structure produced by the capillaries.

Our numerical method is based on a boundary integral formulation. Since the pioneer work by Longuet-Higgins and Cokelet, ${ }^{15}$ boundary integral methods have been popular in computing this type of interfacial problems. However, these methods are known to be very sensitive to numerical instabilities. ${ }^{16}$ The inclusion of surface tension effects makes the discretizations even more sensitive, as was observed 
early by Pullin. ${ }^{17}$ Some local average technique has been used to suppress the numerical instability. This only delays the appearance of the instability, but does not completely eliminate its source. The computations will break down eventually due to the instability. Other techniques such as redistribution and point insertion of vortices ${ }^{18}$ aimed to control the numerical instabilities introduce too much smoothing. This makes it difficult to capture the true small-scale effects associated with surface tension. In addition, surface tension imposes a severe time-step stability constraint (stiffness) for explicit schemes. As a result of all these difficulties, there has been lack of detailed numerical investigation for unsteady breaking waves with surface tension.

Considerable progress has been achieved in the last few years in the analysis and design of robust boundary integral methods. In Ref. 19, Beale, Hou, and Lowengrub have performed a systematic stability analysis of a boundary integral method for two-dimensional (2-D) water waves. They found that there is a compatibility between the choice of the quadrature rule for the singular velocity integral and the choice of the spatial derivative. This compatibility ensures that a delicate balance of terms on the continuous level is preserved on the discrete level. This balance is crucial for maintaining numerical stability. Based on this analysis, they designed a boundary integral method for 2-D water waves with and without surface tension and proved rigorously its convergence in a time continuous framework. Surface tension also introduces additional difficulty in the time discretization. High-order spatial derivatives and Lagrangian particle clustering induce a severe time-step stability constraint. Based on a reformulation using the equal arclength and tangent angle variables, an efficient implicit discretization was proposed by Hou, Lowengrub, and Shelley ${ }^{20}$ which removes this severe time-step stability constraint. The method has been successfully applied to compute with high accuracy a rich variety of complex long-time motion of vortex sheets. ${ }^{21}$ The convergence of this method has been established by Ceniceros and $\mathrm{Hou}^{22}$ for a general two-fluid interface. The stability of boundary integral methods for vortex sheets with surface tension has also been investigated by Baker and Nachbin ${ }^{23}$ based on Fourier analysis near equilibrium solutions.

In this paper, we perform a numerical study of breaking waves using the nonstiff and spectrally accurate boundary integral method developed in Ref. 20. We find that the capillary waves originate near the crest in a neighborhood where both the curvature and its derivative are maximum. For fixed but small surface tension, the maximum of curvature increases in time and the interface develops a train of capillary waves in the forward front of the crest. Our numerical experiments also show that the minimum distance between adjacent capillary crests appears to approach zero, suggesting the formation of trapped bubbles as observed by Koga in experiments of breaking waves. ${ }^{24}$ On the other hand, for a fixed time, as the surface tension coefficient $\tau$ is reduced, both the capillary wavelength and its amplitude decrease nonlinearly. The corresponding waves approach the $\tau=0$ profile. At the onset of the capillaries, the derivative of the convection is comparable to that of the gravity term in the dynamic boundary condition and the surface tension becomes appreciable with respect to these two terms. We find that, based on the $\tau=0$ wave, it is possible to estimate a threshold value $\tau_{0}$ such that if $\tau \leqslant \tau_{0}$ then no capillary waves arise. For $\tau$ sufficiently large, breaking is inhibited and pure capillary motion is observed. The limiting behavior is very similar to that in the classical KdV equation.

We also consider how the viscosity may affect the generation of capillary waves. To this end, we incorporate weak viscous effect into our boundary integral formulation using a boundary layer approach due to Lundgren and Mansour. ${ }^{25}$ This amounts to modifying the dynamic boundary condition at the interface. Lundgren and Mansour observed that one of the terms introduced by the viscous layer correction induces a strain that leads to severe point clustering and numerical instability. Our implicit scheme based on the equal arclength and tangent angle formulation automatically eliminates this difficulty. Our numerical study shows that the interface is smoother but that capillary waves can still appear in this case provided the viscosity is sufficiently small.

The rest of the paper is organized as follows. In Sec. II we present the equations of motion in a boundary integral formulation for 2-D water waves. Our stable boundary integral method is given in Sec. III. This is followed by a description of of the modified equations, which include the weak viscous effects (Sec. IV). The numerical experiments are presented and discussed in Sec. V. The conclusions are given in Sec. VI.

\section{THE EQUATIONS OF MOTION}

We consider one layer of incompressible, irrotational, and inviscid fluid of infinite depth in two dimensions. The position of the fluid interface, at any instant, is given by the complex variable $z(\alpha, t)=x(\alpha, t)+i y(\alpha, t)$, with $\alpha$ a Lagrangian parameter. We further assume that $z(\alpha)-\alpha$ is $2 \pi$ periodic. Then, the complex velocity $W=U-i V$ at the interface can be expressed in terms of a boundary integral as follows (for a derivation see Refs. 26 and 19):

$$
\begin{aligned}
W(\alpha, t)= & \frac{\gamma(\alpha, t)}{2 z_{\alpha}(\alpha, t)}+\frac{1}{4 \pi i} \int_{0}^{2 \pi} \gamma\left(\alpha^{\prime}, t\right) \\
& \times \cot \frac{1}{2}\left[z(\alpha, t)-z\left(\alpha^{\prime}, t\right)\right] d \alpha^{\prime},
\end{aligned}
$$

where the subscript $\alpha$ denotes differentiation with respect to that variable and $\gamma$ is the (unnormalized) vortex sheet strength, which is linked to the velocity potential $\phi$ via the integral equation

$$
\begin{aligned}
\phi_{\alpha}(\alpha)= & \frac{1}{2} \gamma(\alpha)+\operatorname{Re}\left(\frac{z_{\alpha}(\alpha)}{4 \pi i} \int_{0}^{2 \pi} \gamma\left(\alpha^{\prime}\right)\right. \\
& \left.\times \cot \frac{1}{2}\left[z(\alpha)-z\left(\alpha^{\prime}\right)\right] d \alpha^{\prime}\right) .
\end{aligned}
$$

Here we have dropped the time variable to simplify the notation.

The effect of surface tension introduces a jump in the pressure across the interface. This jump is proportional to the 
local mean curvature. Since vacuum is assumed above the water surface, the dynamic boundary condition takes the form of the Bernoulli equation, which, in Eulerian variables, reads as

$$
\left.\frac{\partial \phi}{\partial t}\right|_{z}+\frac{1}{2}|W|^{2}=-g y+\tau \kappa
$$

where $g$ is the gravity acceleration and $\tau$ is the surface tension coefficient. Here, the equations have been nondimensionalized on a periodicity length $\lambda$ (so that the nondimensional wavelength is 1) and a velocity scale $\bar{\gamma}$ equal to the average of $\gamma$ over one period. Note that $\bar{\gamma} / 2$ gives the velocity at $y=-\infty$. Thus, $\tau=\widetilde{\tau} /\left(\bar{\gamma}^{2} \lambda\right)$ and $g=\tilde{g} \lambda / \bar{\gamma}^{2}$, where $\widetilde{\tau}$ and $\tilde{g}$ are the dimensional surface tension and gravity coefficients, respectively. The curvature $\kappa$ is given by

$$
\kappa=\frac{y_{\alpha \alpha} x_{\alpha}-x_{\alpha \alpha} y_{\alpha}}{\left(x_{\alpha}^{2}+y_{\alpha}^{2}\right)^{3 / 2}} .
$$

We define the velocity $\widetilde{W}$ of the Lagrangian particles on the interface as

$$
\widetilde{W}=W+\frac{z_{\alpha}^{*}}{\left|z_{\alpha}\right|} U^{A}
$$

where the asterisk stands for the complex conjugate. With this velocity, we still satisfy the kinematics and have freedom to select $U^{A}$ to gain some control over the positioning of the Lagrangian particles. Thus,

$$
\frac{\partial z}{\partial t}(\alpha, t)=\widetilde{W}^{*}(\alpha, t) .
$$

The Bernoulli equation can be evaluated in this Lagrangian frame, by noting that

$$
\left.\frac{\partial \phi}{\partial t}\right|_{\alpha}=\left.\frac{\partial \phi}{\partial t}\right|_{z}+\operatorname{Re}\left\{W^{*} \tilde{W}\right\}=\left.\frac{\partial \phi}{\partial t}\right|_{z}+|W|^{2}+U^{A} U^{T},
$$

where $U^{T}=\left|z_{\alpha}\right|^{-1} \operatorname{Re}\left\{z_{\alpha} W\right\}$. Hence, we obtain

$$
\left.\frac{\partial \phi}{\partial t}\right|_{\alpha}=\frac{1}{2}|W|^{2}+U^{A} U^{T}-g y+\tau \kappa .
$$

Equations (1)-(4) determine the time evolution of the interface. Note that it is $\phi_{\alpha}$, not $\phi$, that is needed to obtain $\gamma$ and the velocity. The evolution equation for $\phi_{\alpha}$ can be obtained by differentiating the Bernoulli equation with respect to $\alpha$. Therefore, it is the competition between $\tau \kappa_{\alpha}$ and $\left(\frac{1}{2}|W|^{2}+U^{A} U^{T}-g y\right)_{\alpha}$ that plays a role in the generation of capillary waves.

A natural variable for the curvature is the tangent angle $\theta$ to the interface, that is $z_{\alpha}=\sigma e^{i \theta}$, where $\sigma$ is the arclength metric $\left|z_{\alpha}\right|$. The curvature has a simple expression in these variables: $\kappa=\theta_{\alpha} / \sigma$. Given $(\sigma, \theta)$, the interface can be reconstructed up to a translation constant (for details see Refs. 22 and 20).

One can easily derive the equations of motion for these two new variables. They are given by

$$
\sigma_{t}=\left(U^{T}+U^{A}\right)_{\alpha}-\theta_{\alpha} U^{N},
$$

$$
\theta_{t}=\frac{U_{\alpha}^{N}}{\sigma}+\frac{\theta_{\alpha}}{\sigma}\left(U^{T}+U^{A}\right)
$$

where $U^{N}=-\left|z_{\alpha}\right|^{-1} \operatorname{Im}\left\{z_{\alpha} W\right\}$ is the normal velocity at the interface. To have better control on the tangential motion of the Lagrangian particles, we select $U^{A}$ such that the following condition is satisfied:

$$
\sigma(\alpha, t)=\frac{1}{2 \pi} \int_{0}^{2 \pi} \sigma\left(\alpha^{\prime}, t\right) d \alpha^{\prime} \equiv \bar{\sigma} .
$$

It is easy to show that this condition is enforced if $U^{A}$ is given by

$$
U^{A}=-U^{T}+\int_{0}^{\alpha}\left[\theta_{\alpha} U^{N}-\overline{\theta_{\alpha} U^{N}}\right] d \alpha^{\prime},
$$

where $\overline{\theta_{\alpha} U^{N}}$ is the spatial average over one period, as defined in (7). Condition (7) gives a uniform distribution of particles on the interface. Since $\sigma$ is now constant in $\alpha$, semiimplicit time discretizations become easy to implement. With this choice of $U^{A}$, the evolution equation for $\sigma$ is the following ordinary differential equation:

$$
\frac{d \sigma}{d t}=-\overline{\theta_{\alpha} U^{N}}
$$

\section{THE NUMERICAL METHOD}

\section{A. A stable space discretization}

Our discretization is based on the pseudospectral approximation for the space derivatives and some careful dealiasing filtering. The singular velocity integral is approximated by the alternate-point trapezoidal rule. This gives a spectrally accurate approximation.

Numerical filtering is defined in Fourier space as follows:

$$
\widehat{\left(f^{p}\right)_{k}}=\rho(k h) \hat{f}_{k},
$$

where the overcaret stands for the discrete Fourier transform and $\rho$ is a non-negative even cutoff function such that $\rho(x)$ $=1$ for $|x| \leqslant \lambda \pi$ with $0<\lambda<1$ and decays smoothly to zero at $x= \pm \pi$. Here $h=2 \pi / N$ with $N$ even. Denoting by $S_{h}$ the spectral derivative operator and setting $\mathrm{D}_{\mathrm{h}} f_{i}=\mathrm{S}_{\mathrm{h}} f_{i}^{p}$, we have the following discretization:

$$
\begin{aligned}
& \frac{d \sigma}{d t}=-\frac{1}{N} \sum_{j=1}^{N} \mathrm{~S}_{\mathrm{h}} \theta_{j} U_{j}^{N}, \\
& \frac{d \theta_{i}}{d t}=\frac{1}{\sigma_{i}}\left[\mathrm{~S}_{\mathrm{h}} U_{i}^{N}+\mathrm{D}_{\mathrm{h}} \theta_{i}\left(U_{i}^{T}+U_{i}^{A}\right)\right], \\
& \frac{d \phi_{i}}{d t}=\frac{\tau}{\sigma_{i}} \mathrm{D}_{\mathrm{h}} \theta_{i}^{p}+\frac{1}{2}\left|W_{i}\right|^{2}+U_{i}^{A} U_{i}^{T}-g \operatorname{Im}\left\{z_{i}\right\} .
\end{aligned}
$$

Given $\sigma$ and $\theta_{i}$, we can reconstruct $z_{i}$ (for details, see Ref. 20). The velocity is computed using the spectrally accurate alternate-point trapezoidal rule for the periodic kernel: 


$$
W_{i}=\frac{\gamma_{i}}{2 \sigma_{i}} e^{-i \theta_{i}^{p}}+\frac{1}{4 \pi i} \sum_{\substack{j=-N / 2+1 \\(j-i) \text { odd }}}^{N / 2} \gamma_{j} \cot \frac{1}{2}\left(z_{i}^{p}-z_{j}^{p}\right) 2 h
$$

At every time step the vortex sheet strength $\gamma$ is obtained by solving, via fixed point iteration, the following discrete integral equation:

$$
\begin{aligned}
\mathrm{D}_{\mathrm{h}} \phi_{i}= & \frac{1}{2} \gamma_{i} \\
& +\operatorname{Re}\left(\frac{\sigma_{i} e^{i \theta_{i}^{p}}}{4 \pi i} \sum_{\substack{j=-N / 2+1 \\
(j-i) \text { odd }}}^{N / 2} \gamma_{j} \cot \frac{1}{2}\left(z_{i}^{p}-z_{j}^{p}\right) 2 h\right) .
\end{aligned}
$$

It has been shown in Ref. 3 that the Neumann series converges globally. As remarked before, $\mathrm{D}_{\mathrm{h}} \phi_{i}$ is evolved in time by applying $D_{h}$ to Eq. (13).

Finally, the tangential and normal velocities are computed from $W_{i}$ as follows:

$$
\begin{aligned}
& U_{i}^{T}=\operatorname{Re}\left\{e^{i \theta_{i}^{p}} W_{i}\right\} ; \quad U_{i}^{N}=-\operatorname{Im}\left\{e^{i \theta_{i}^{p}} W_{i}\right\}, \\
& U_{i}^{A}=-U_{i}^{T}+\operatorname{Int}_{\mathrm{h}}\left(\mathrm{D}_{\mathrm{h}} \theta U^{N}-\frac{1}{N} \sum_{j=1}^{N} \mathrm{D}_{\mathrm{h}} \theta_{j} U_{j}^{N}\right) i .
\end{aligned}
$$

Here $\mathrm{Int}_{\mathrm{h}}$ stands for the pseudospectral approximation to integration with the zeroth mode set to zero.

Following the lines of the proof given in Ref. 6, it can be shown that this discretization yields a convergent spectral method for sufficiently smooth solutions. ${ }^{27}$

\section{B. An efficient time discretization}

To achieve an efficient time discretization without the high-order time-step constraint introduced by the surface tension, we perform a small-scale decomposition as done by Hou, Lowengrub, and Shelley ${ }^{20}$ to separate the terms contributing to the stiffness in the equations of motion.

The dominant small-scale terms are the curvature in the Bernoulli equation and the derivative of the normal velocity in the evolution equation for $\theta$. It can be shown that ${ }^{20}$

$$
U_{i}^{N}=\frac{1}{2 \sigma} \mathrm{H}_{\mathrm{h}} \gamma_{i}+\mathrm{R}_{\mathrm{h}} \gamma_{i}
$$

where

$$
\mathrm{H}_{\mathrm{h}} f_{i}=\frac{1}{2 \pi} \sum_{\substack{j=-N / 2+1 \\(j-i) \text { odd }}}^{N / 2} f_{j} \cot \frac{1}{2}\left(\alpha_{i}-\alpha_{j}\right) 2 h
$$

is a discrete version of the Hilbert transform for periodic functions and $\mathrm{R}_{\mathrm{h}} \gamma_{i}$ is a smoother term. Also, from the integral equation for $\gamma_{i}$ we have that, to leading order $\gamma_{i}$ $\sim 2 \mathrm{D}_{\mathrm{h}} \phi_{i}$. Therefore, the discrete evolution equations can be written as

$$
\frac{d \theta_{i}}{d t}=\frac{1}{\sigma^{2}} \mathrm{H}_{\mathrm{h}} \mathrm{S}_{\mathrm{h}}^{2} \phi_{i}^{p}+P_{i},
$$

$$
\frac{d \phi_{i}}{d t}=\frac{\tau}{\sigma} \mathrm{D}_{\mathrm{h}} \theta_{i}^{p}+Q_{i},
$$

where $P_{i}$ and $Q_{i}$ are lower-order terms at small spatial scales and can be treated explicitly in time. We have also used the fact that $\sigma$ is constant in $\alpha$.

With this decomposition, semi-implicit time integration methods are straightforward and easy to implement. The implicit terms can be inverted fast in Fourier space. Here, we employ the fourth-order multistep implicit/explicit scheme developed by Ascher, Ruuth, and Wetton. ${ }^{28}$

\section{WEAK VISCOUS EFFECTS}

In this section we present the modified equations to incorporate weak viscous effects into our boundary integral formulation for water waves. The basic idea from Lundgren and Mansour ${ }^{25}$ is the assumption that viscous effects for free-surface problems are confined to thin weak vortical boundary layers. The viscous correction can be expressed completely in terms of the interior potential flow as a modification to the boundary conditions. Here we only give the equations. Their derivation can be obtained following Refs. 29 and 25.

The modified evolution equations are

$$
\begin{aligned}
z_{t}^{*}= & W+\frac{z_{\alpha}^{*}}{\left|z_{\alpha}\right|}\left(U^{A}-i u^{N}\right) ; \quad u^{N}=-\frac{\psi_{\alpha}}{\left|z_{\alpha}\right|}, \\
\psi_{t}= & -\frac{2 \psi}{\left|z_{\alpha}\right|} \operatorname{Re}\left\{z_{\alpha} W_{\alpha}\right\}-\frac{2 \nu}{\left|z_{\alpha}\right|} \operatorname{Im}\left\{z_{\alpha} W_{\alpha}\right\}-u^{N} U^{A}, \\
\phi_{t}= & \tau \kappa+\frac{1}{2}|W|^{2}+U^{T} U^{A}-g y+u^{N} U^{N} \\
& +\frac{2 \psi}{\left|z_{\alpha}\right|} \operatorname{Im}\left\{z_{\alpha} W_{\alpha}\right\}+\frac{2 \nu}{\left|z_{\alpha}\right|} \operatorname{Re}\left\{z_{\alpha} W_{\alpha}\right\},
\end{aligned}
$$

where $W$ is again the complex velocity given by (1), $\psi$ is the streamfunction, and $\nu$ is the viscosity coefficient (nondimensionalized with length scale $\lambda$ and velocity scale $\bar{\gamma}$ ). These equations are an asymptotic approximation to order $O\left(\nu^{3 / 2}\right)$
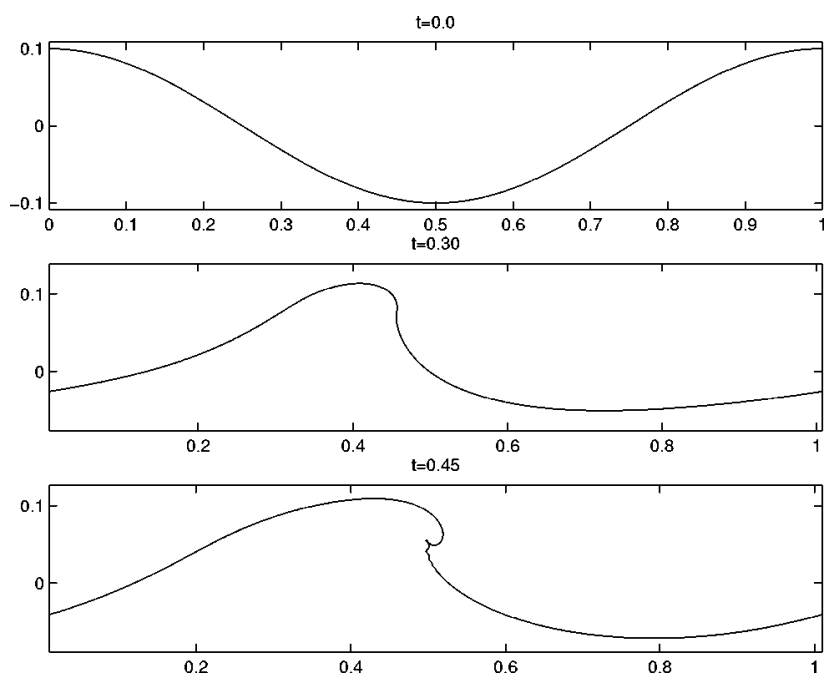

FIG. 1. Breaking wave profiles for $\tau=0.001$ at times $t=0,0.30$, and 0.45 . Here $N=2048$ and $\Delta t=5 \times 10^{-5}$. 

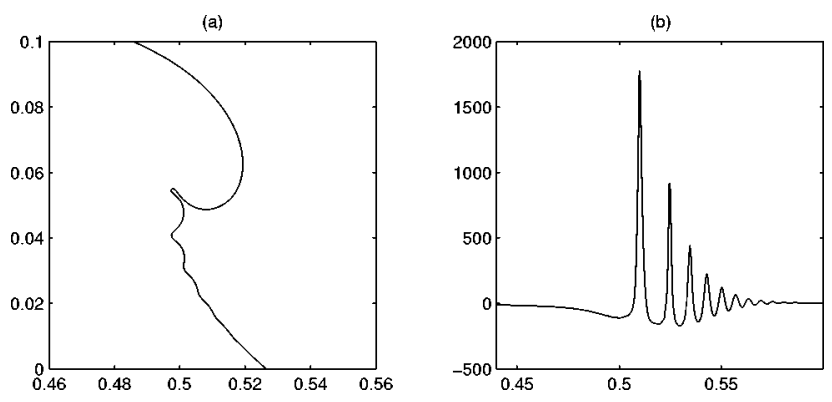

FIG. 2. (a) A close-up of the interface at $t=0.45$ and (b) the curvature plotted against the Lagrangian parameter $\alpha$ for $\tau=0.001, N=2048$, and $\Delta t=5 \times 10^{-5}$.

or to third order in the vortical layer width $\delta=O(\sqrt{\nu})$. To recast the equations in the $(\sigma, \theta)$ variables, we just need to change the normal velocity to $u^{N}+U^{N}$.

\section{NUMERICAL EXPERIMENTS}

\section{A. Inviscid waves}

We begin our numerical experiments with a simulation using the method (11)-(17). The computations are for 1-periodic interfaces; this was done by a simple scaling of the $2 \pi$-periodic case. All the simulations presented here use the following initial data:

$$
\begin{aligned}
& z(\alpha, 0)=\alpha+0.1 i \cos (2 \pi \alpha), \\
& \gamma(\alpha, 0)=-1+0.1 \sin (2 \pi \alpha) .
\end{aligned}
$$

Note that the vortex sheet strength $\gamma$ has a nonzero mean $(\bar{\gamma}=-1)$. This gives the wave an impulse that eventually cause it to overturn. Here, $\bar{\gamma} / 2$ gives the velocity at $y$ $=-\infty$. We have computed previously with other initial conditions $(\bar{\gamma}=0)$ and finite depth in Ref. 27, where we also found that the capillary waves appear once the interface steepens up and is about to overturn. However, since finite depth computations are considerably more expensive than those for deep water, we cannot achieve the high resolution needed to compute the subsequent development of the capillary waves and their detailed structure, as we are able to show in the deep water computations presented below.

We take $g=10$ and vary the surface tension coefficient $\tau$ throughout the experiments. Our first simulation is for $\tau$ $=0.001$. In Fig. 1, we present the interface profile at times $t=0,0.30$, and 0.45 , computed using $N=2048$ and $\Delta t=5$ $\times 10^{-5}$. The interface becomes vertical at $t=0.30$ and a cap-
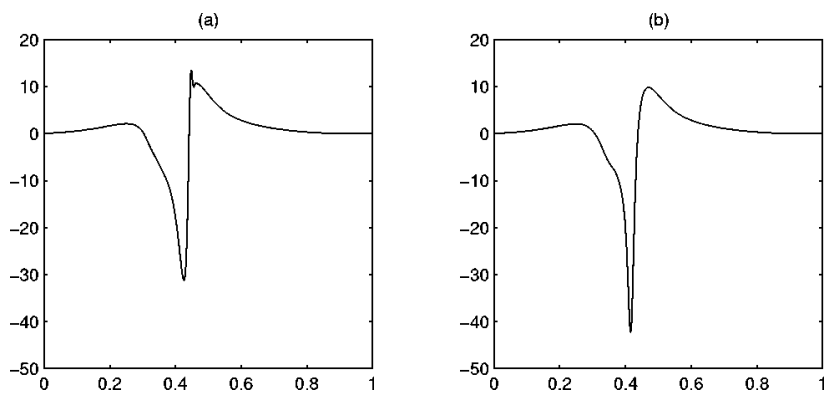

FIG. 3. Curvature versus $\alpha$ at $t=0.26$. (a) $\tau=0.001$ and (b) $\tau \rightarrow 0$.
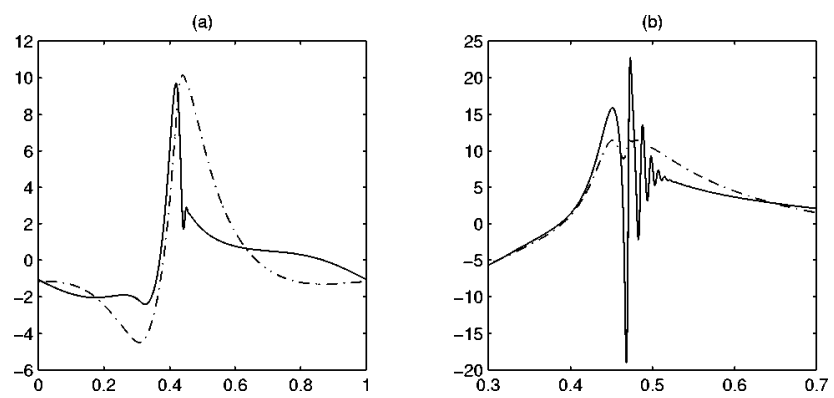

FIG. 4. A comparison of $\left(\frac{1}{2}|W|^{2}+U^{A} U^{T}\right)_{\alpha}$ (solid line) and $-g y_{\alpha}$ (dotted line) for $\tau=0.001$ at (a) $t=0.25$ and (b) $t=0.35$.

illary wave appears soon after the wave begins to overturn. Figure 2(a) gives a close-up of a neighborhood of the crest at time $t=0.45$. The curvature for that region is plotted in Fig. 2(b), where a capillary wave train is clearly observed.

Although the interface appears very smooth at $t=0.30$, the onset of the capillary waves occurs before that time, as a plot of the curvature at $t=0.26$ shows [Fig. 3(a)]. Note that there is a spike at $\alpha=0.45$, right after the wave tip ( $\alpha$ $=0.43$ ). Figure 3(b) shows that such a spike is not present in the case $\tau=0$, where the curvature is smoother at $\alpha=0.43$ but larger at the tip, as expected. Near the time of onset of the capillaries (about $t=0.25$ ) the convection term $\left(\frac{1}{2}|W|^{2}\right.$ $\left.+U^{A} U^{T}\right)_{\alpha}$ is comparable to the gravity term $-g y_{\alpha}$, as Fig. 4(a) demonstrates. Note that the effect of surface tension is already visible in the convection term in the form of an inverted spike at the toe $(\alpha=0.43)$. Figure 4(b) compares these two terms at a subsequent time $(t=0.35)$.

A closer look at the interface profiles near $t=0.45$ [Fig. 5 (a)] shows that the capillary trough gets narrower, suggesting a possible formation of a trapped bubble through selfintersection of the interface. The minimum distance between adjacent parts of the interface near the "edge" of the capillary wave is decreasing in time [Fig. 5(b)]. On the other hand, the tangent angle $\theta$ becomes nearly discontinuous with
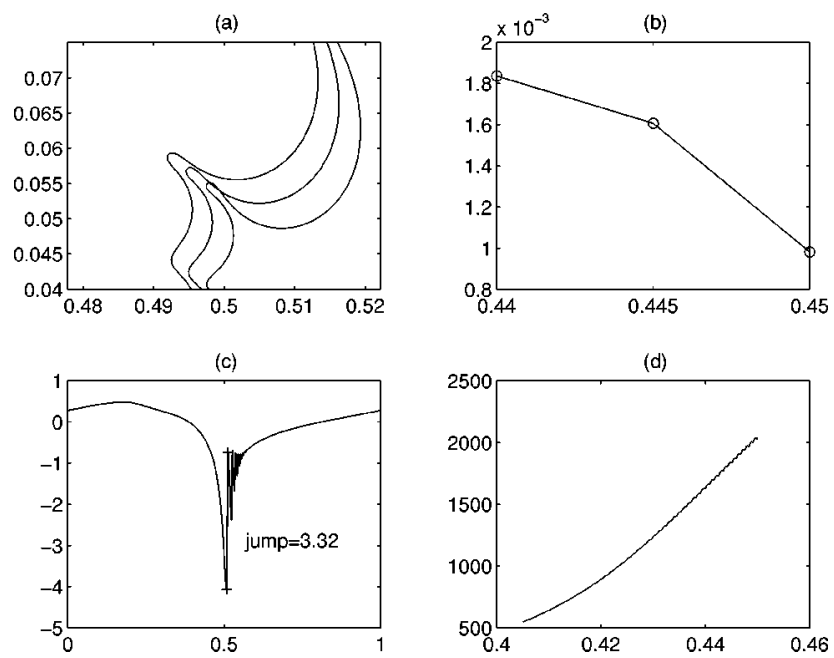

FIG. 5. Case $\tau=0.001, N=2048$, and $\Delta t=5 \times 10^{-5}$. (a) Close-ups of the interface at $t=0.44,0.445$, and 0.45 (left to right). (b) The minimum distance between opposite sides of the interface near the edge. (c) Tangent angle $\theta$ vs $\alpha$ at $t=0.45$. (d) The maximum of $|\kappa|$ versus time. 

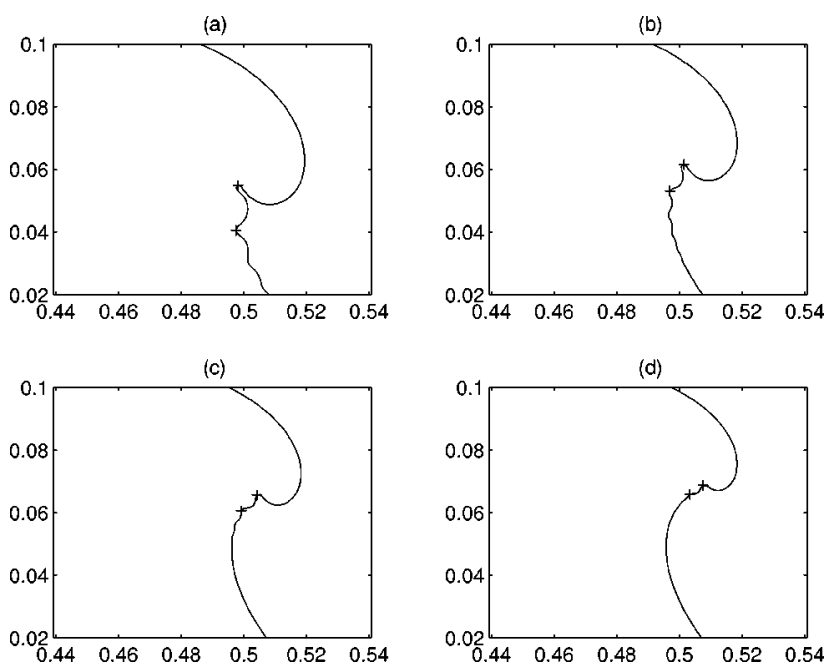

FIG. 6. Interface close-ups at $t=0.45$ for (a) $\tau=0.001$, (b) $\tau=5 \times 10^{-4}$, (c) $\tau=2.5 \times 10^{-4}$, (d) $\tau=1.25 \times 10^{-4}$. The distance between the crosses is $\lambda_{\tau}$.

a jump size close to $\pi$, as in a cusp [Fig. 5(c)]. We remark that both the curvature and its derivative have maxima (in absolute value) in a small neighborhood about the edge. In that region, the curvature continues to grow in time, as it can be seen in Fig. 5(d).

Now, for a fixed time $(t=0.45)$ we compute the capillary wavelength $\lambda_{\tau}$ and the amplitude $a_{\tau}$ as the surface tension coefficient $\tau$ is decreased (Fig. 6). The capillary wavelength $\lambda_{\tau}$ is defined as the the distance between the two largest values of $|\kappa|$. These are the crosses shown in Fig. 7, where close-ups of the capillary region corresponding to different $\tau^{\prime}$ s are presented. The behavior in time of $\lambda_{\tau}$ and $a_{\tau}$ for $\tau=0.001$ is given in Fig. 8. While the capillary wavelength $\lambda_{\tau}$ has a relatively small change over the time interval $[0.40,0.45]$, the capillary amplitude $a_{\tau}$ increases more rapidly. Nevertheless, $a_{\tau}$ remains fairly small, even at $t=0.45$.

Although it is difficult to obtain a scaling for $\lambda_{\tau}$ and $a_{\tau}$, it is clear from Fig. 6 that both quantities decrease nonlinearly as $\tau$ decreases. Therefore, we can expect that the limiting solutions converge strongly to the $\tau=0$ profile for a fixed time. This indeed appears to be the case, as observed in Fig. 9, where the surface tension coefficient $\tau$ is decreased even further. We observe the convergence of the corresponding interface profiles to that corresponding to the zero surface tension profile at the fixed time $t=0.45$.
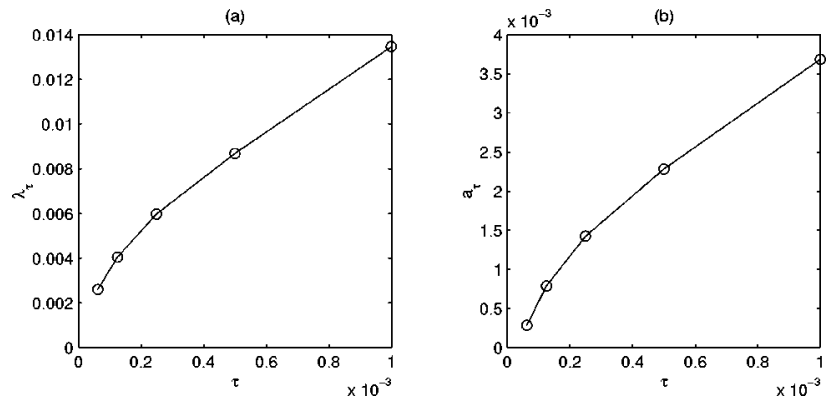

FIG. 7. The computed capillary wavelength $\lambda_{\tau}$ and the amplitude $a_{\tau}$. (a) $\lambda_{\tau}$ vs $\tau$. (b) $a_{\tau}$ vs $\tau$.
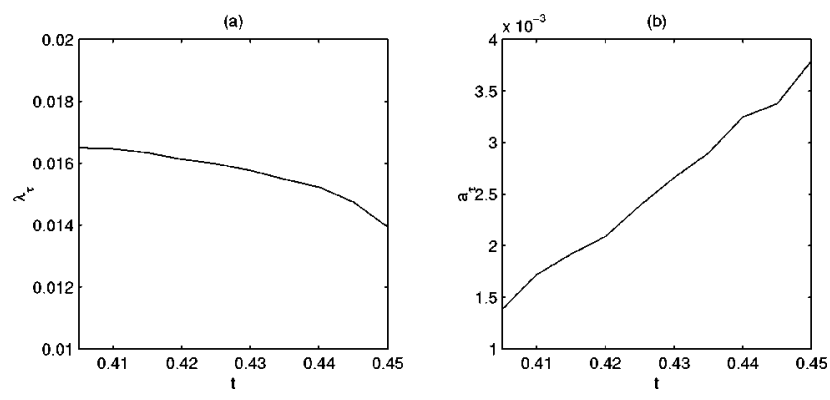

FIG. 8. For $\tau=0.001$, (a) the capillary wavelength $\lambda_{\tau}$ versus time and (b) the capillary amplitude $a_{\tau}$ versus time.

Decreasing $\tau$ delays the onset of the capillary waves until the derivative of the curvature is large enough to excite the surface tension term. More precisely, from the zero surface tension interface we can estimate a bound $\tau_{0}$ such that $\tau_{0} \kappa_{\alpha}$ will be negligible compared to the derivative of the convection and gravity terms in Bernoulli's equation. The comparison plots for these terms are shown in Fig. 10 for the particular initial data $(24)-(25)$ at $t=0.5$. At this time, $\max \left|\kappa_{\alpha}\right|=2.5 \times 10^{4}$. Therefore, if we take $\tau=1 /\left(\max \left|\kappa_{\alpha}\right|\right)$ $=4 \times 10^{-5}$, the term $\tau \kappa_{\alpha}$ would be order 1 and about $0.8 \%$ of the other competing term [Fig. 10(b)]. Although no capillaries are observed for such $\tau$ in the wave profile, the curvature near $t=0.5$ has signs of their presence. We find numerically that if $\tau \leqslant \tau_{0}=1 \times 10^{-6}$, then both the interface and its curvature remain smooth and no capillary waves are observed. Such values of $\tau$ would give a dispersive term $\tau \kappa_{\alpha}$ less than $0.03 \%$ of the maximum value of the derivative of the convection and gravity terms.

On the other extreme if $\tau$ is sufficiently large, then the capillary effects dominate and the wave does not overturn. A typical behavior is shown in Fig. 11 for $\tau=0.5$ for long times.

The intermediate case $\tau=0.1$ is also very interesting (Fig. 12). Now, the front does not overturn but forms a bulge (a)

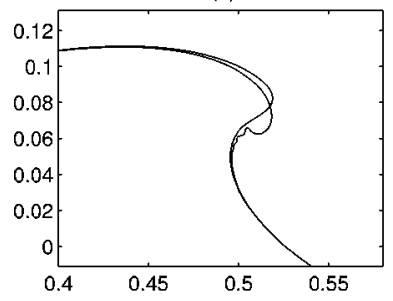

(c)

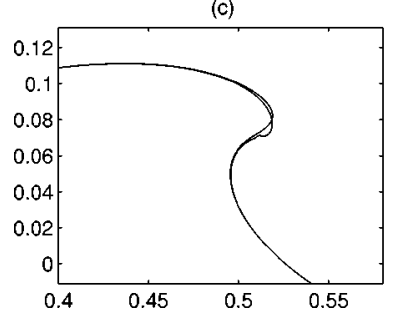

(b)
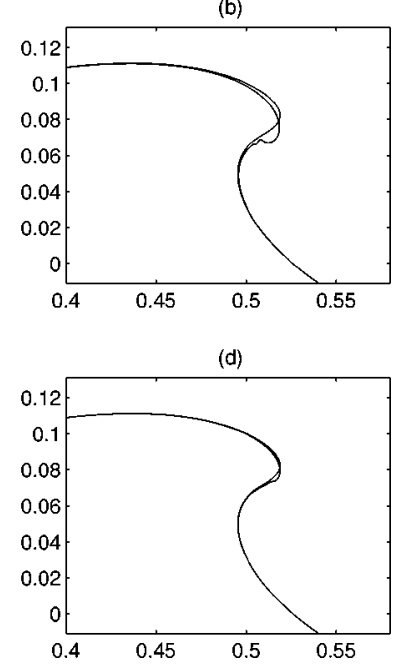

FIG. 9. A comparison of the zero surface tension interface profile with the corresponding ones for decreasing $\tau$ at $t=0.45$. (a) $\tau=2.5 \times 10^{-4}$. (b) $\tau$ $=1.25 \times 10^{-4}$. (c) $\tau=6.25 \times 10^{-5}$. (d) $\tau=3.125 \times 10^{-5}$. 


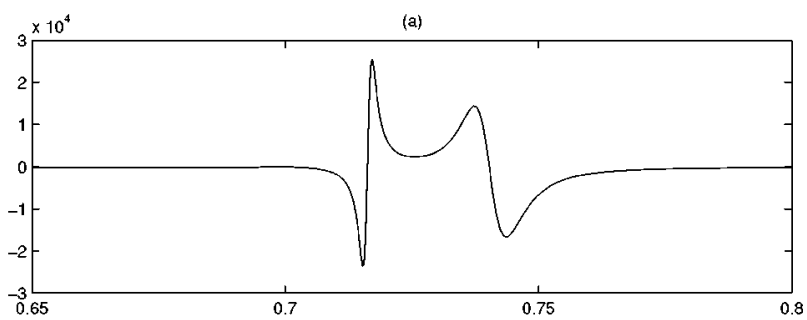

(b)

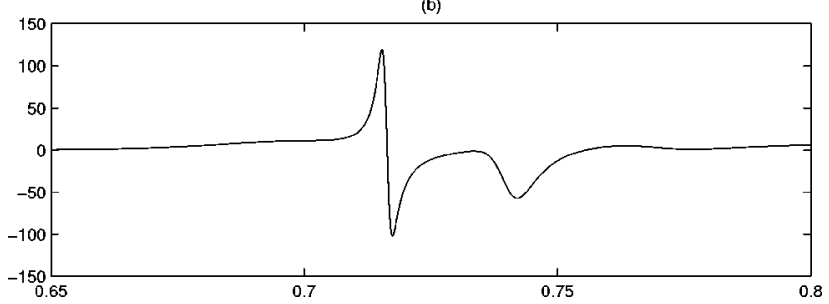

FIG. 10. (a) A derivative of the curvature, $\max \left|\kappa_{\alpha}\right|=2.5 \times 10^{4}$. (b) A derivative of the convection and gravity terms. Both plots correspond to the zero surface tension wave at $t=0.5, N=1024$, and $\Delta t=1.25 \times 10^{-4}$.

that interacts with the capillary waves. Dispersion becomes strong at $t=0.85$ and the delicate competition among convection, gravity, and surface tension appears to eventually lead to the formation of a trapped bubble (Fig. 13).

\section{B. Analogy with the $\mathrm{KdV}$ equation}

Probably the simplest model that has both nonlinear convection and dispersion is the classical $\mathrm{KdV}$ equation,

$$
u_{t}+u u_{x}=\epsilon u_{x x x} .
$$

To illustrate the similarities between the small dispersion solutions to this equation and that of the capillaries on a breaking wave, let us consider the initial data $u_{0}(x)$ $=\sin 2 \pi x$ in the interval $[0,1]$. For $\epsilon=0$, a shock will be formed at the breaking time $t_{b}=1 / 2 \pi$, but the solution $u(t, x ; \epsilon=0)$ is smooth for $t<t_{b}$.

For small but nonzero $\epsilon$, an oscillatory train may appear in the bottom of the forward face of the front. This is illustrated in Fig. 14 in the first two plots of the left column. The
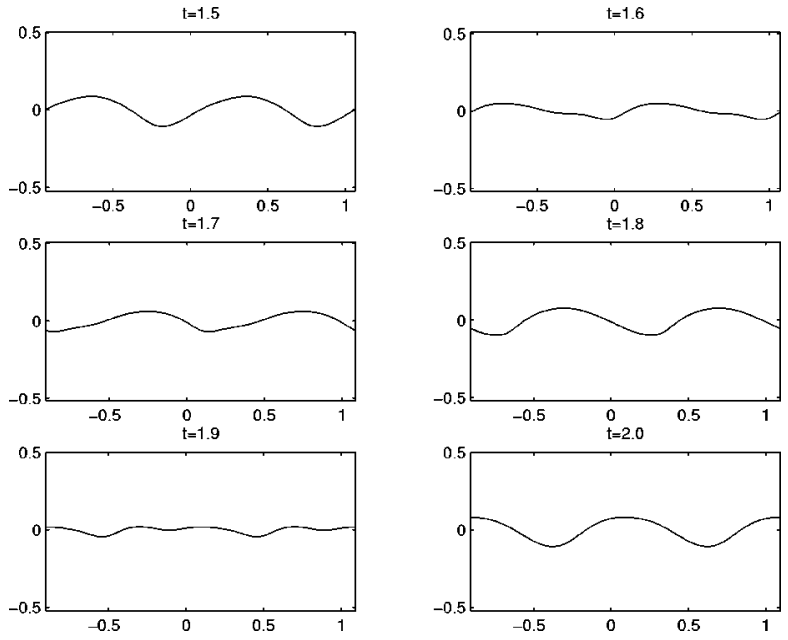

FIG. 11. Interface profiles at times $1.5-2.0$ for $\tau=0.5$. The computation is performed with $N=1024$ and $\Delta t=1.25 \times 10^{-4}$.
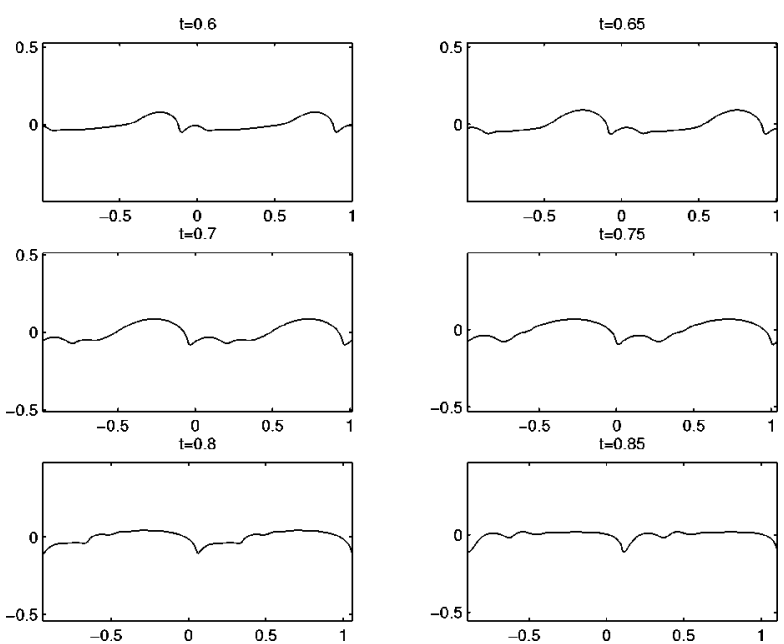

FIG. 12. Interface profiles at times $1.5-2.0$ for $\tau=0.1$. The computation performed with $N=1024$ and $\Delta t=1.25 \times 10^{-4}$.

third derivative of the corresponding solution appears in the right column of this figure. The solutions are plotted at the time $t=0.15<t_{b}$ and were computed using a semi-implicit spectral discretization with $N=1024$ points and $\Delta t=1.25$ $\times 10^{-4}$.

As $\epsilon$ tends to zero, both the wavelength and the amplitude of these oscillations decrease monotonically and the solution $u(t, x ; \epsilon)$ converges strongly to $u(t, x ; 0)$ for $t<t_{b}$. On the other hand, if $\epsilon<\epsilon_{0}=1 \times 10^{-8}$ then no signs of oscillations appear in the solution or its third derivative. Again, the onset of the oscillations is determined by the balance between convection and dispersion. An estimate for $\epsilon_{0}$ may be obtained by considering the maximum of $\left|u_{x x x}(t, x, 0)\right|$. In this case, $1 / \max \left|u_{x x x}(t, x, 0)\right| \sim 5 \times 10^{-8}$. Although with such a value for $\epsilon$ no oscillations appear in the solution, the third derivatives has some signs of dispersion. Taking $\epsilon_{0}=1$ $\times 10^{-8}$ makes the dispersive term effectively negligible (plots in the bottom row in Fig. 14). Clearly, the behavior of the small dispersion solutions of the $\mathrm{KdV}$ equation is very similar to the more complex motion of breaking water waves with small surface tension.
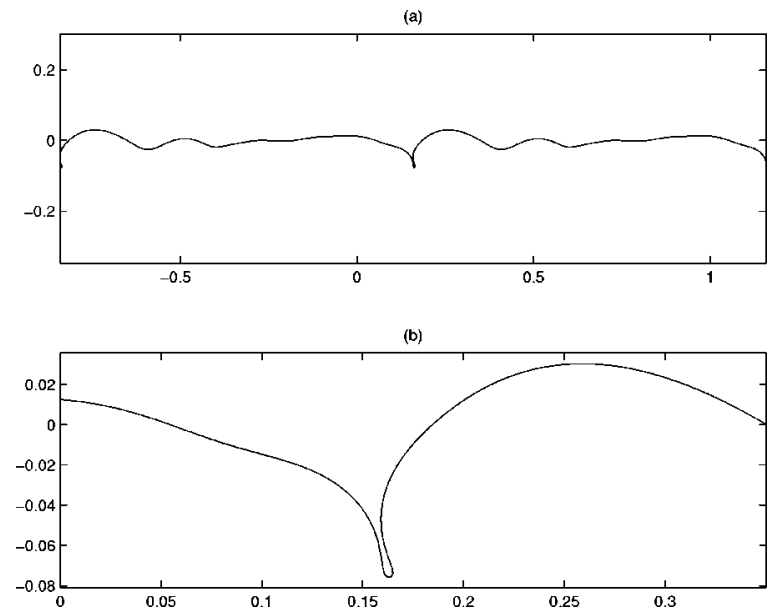

FIG. 13. (a) An interface at time 0.896 for $\tau=0.1$. (b) Close-up. $N=1024$ and $\Delta t=1.25 \times 10^{-4}$. 

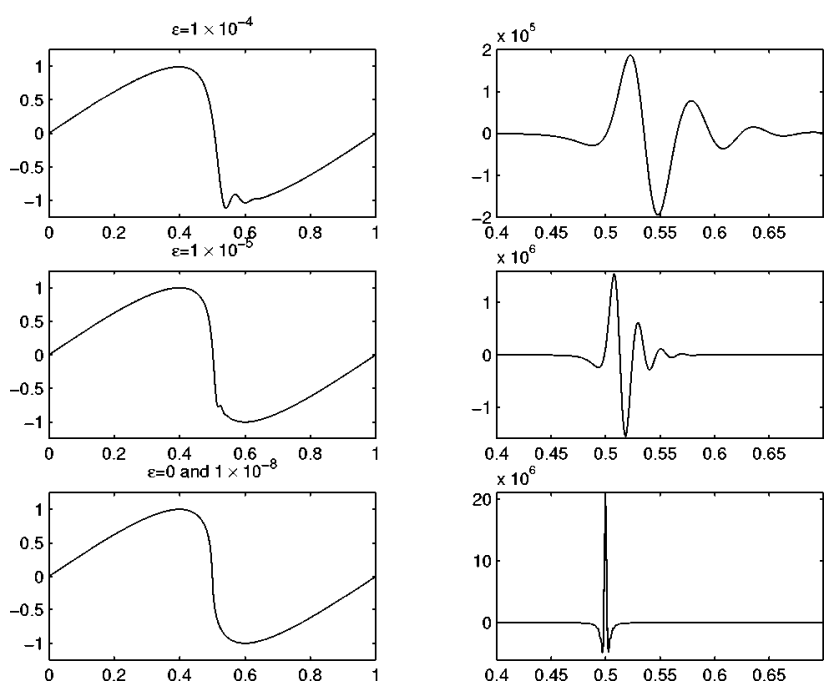

FIG. 14. The plots on the left show the solution $u(x)$ of the KdV equation for different $\epsilon$ at $t=0.15$. The plots on the right show their corresponding $u_{x x x}$.

\section{Weak viscous effects}

We illustrate the weak viscous effects by two numerical examples of a breaking wave. The weak viscous effect is modeled by the equations presented in Sec. IV. Both examples use the same initial data and gravity coefficient as before. In the first example, we include both surface tension and the weak viscosity contribution. We take $v=0.0005$ and $\tau=0.001$. A sequence of the wave profiles is shown in Fig. 15. For this set of parameters, the viscous effects dominate the surface tension. There are no signs of capillary waves, as a closer look at the profile and curvature shows in Fig. 16. The curvature varies smoothly and its maximum is only about $10 \%$ of that corresponding to $\nu=0$ and with $\tau$ $=0.001$.

However, capillary waves are observed for a smaller viscosity coefficient (Fig. 17). Again, the capillary wave can be seen more clearly as the oscillations in the curvature corre-
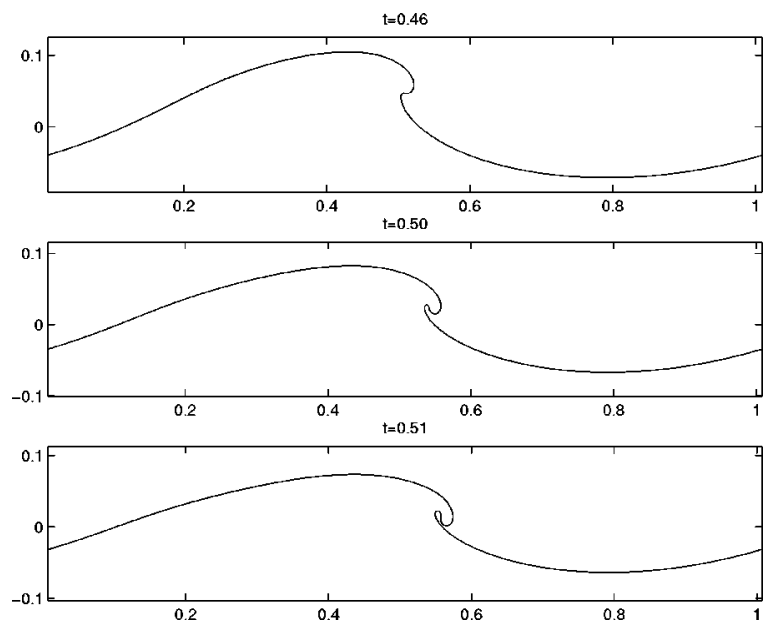

FIG. 15. Wave profiles for $\nu=0.0005$ and $\tau=0.001$ at times $t=0.46,0.50$, and 0.5 . Here $N=1024$ and $\Delta t=1.25 \times 10^{-4}$.
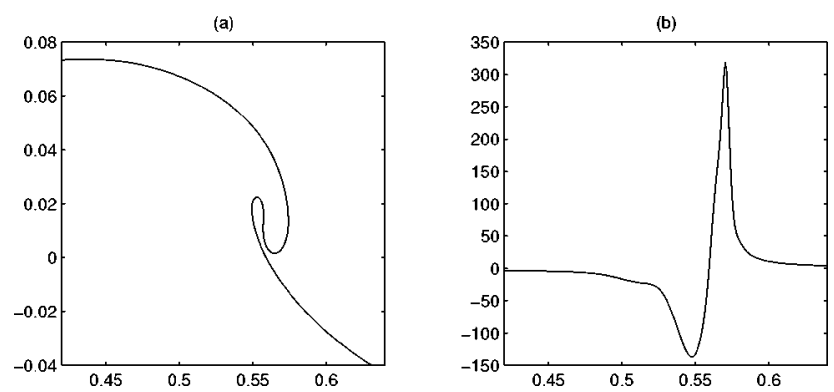

FIG. 16. (a) Interface at time $t=0.51$ for $\nu=0.0005$ and $\tau=0.0001$. (b) Curvature at the same time. Here $N=1024$ and $\Delta t=1.25 \times 10^{-4}$.

sponding to the forward side of the wave [Fig. 17(b)]. The simulation also suggests the formation of a trapped bubble in finite time.

\section{CONCLUSIONS}

We have shown through highly accurate numerical simulations the dynamic generation of capillary waves on the surface of breaking water waves. The capillaries originate near the wave crest in a neighborhood where both the curvature and its derivative are maximum. It is the derivative of the curvature, competing with convection and gravity terms, that plays a role in the onset of the capillaries. This is similar to the onset of dispersive waves in the classical KdV equation before the formation of a shock.

Our numerical experiments also show that both the capillary wavelength and its amplitude decrease nonlinearly as the surface tension coefficient $\tau$ tends to zero and, therefore, the wave profiles converge strongly to the $\tau=0$ wave profile for a fixed time. Moreover, based on the zero surface tension wave, an estimated value $\tau_{0}$ can be found such that no capillaries are observed if $\tau<\tau_{0}$ for the given initial data.

The robust and efficient numerical method we used, allowed us to consider a wide range of surface tension coefficients. Smooth capillary dominant behavior was observed for large values of $\tau$, while intermediate and small values appear to eventually lead to the formation of trapped bubbles, which constitutes a topological singularity of the interface.

Weak viscous effects were also incorporated into our boundary integral method. The numerical examples pre-
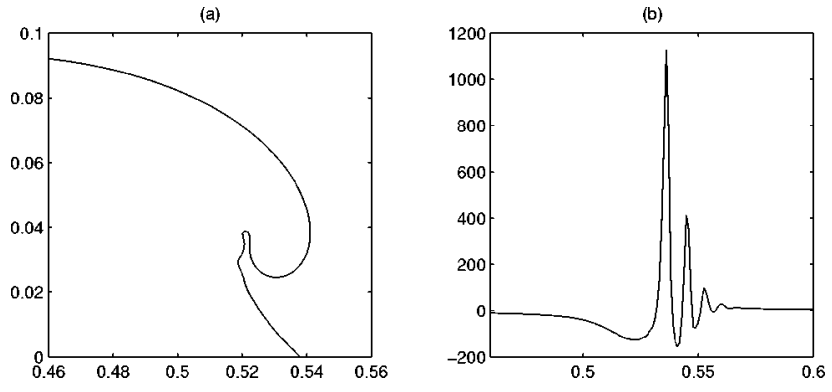

FIG. 17. (a) A close-up of the interface at time $t=0.48$ for $\nu=0.0001$ and $\tau=0.0001$. (b) Curvature at the same time. Here $N=1024$ and $\Delta t=1.25$ $\times 10^{-4}$. 
sented here showed that the wave profile is smoother but capillary waves may still appear if the viscosity coefficient is sufficiently small.

We remark finally, that although the curvature near the capillary edge appears to grow monotonically in time, a higher resolution study is needed to determine whether it (or its derivative) will blow up in finite time and if so at what rate. It would also be very interesting to study further the possible topological singularity associated with trapped bubbles, as it is believed to precede a turbulent wave breaking, as suggested by the experiments of Duncan et al. ${ }^{1}$

\section{ACKNOWLEDGMENTS}

We would like to thank Professor Bjorn Engquist, Professor Phillip Saffman, and Professor Ted Wu for many helpful discussions regarding this work. We would also like to thank the referees for their suggestions and comments. The research was supported in part by an Office of Naval Research grant No. N00014-96-1-0438 and by a National Science Foundation grant No. DMS-9704976.

${ }^{1}$ J. H. Duncan, V. Philomin, M. Behres, and K. Kimmel, "The formation of spilling breaking water waves,' Phys. Fluids 6, 2558 (1994).

${ }^{2}$ N. Ebuchi, H. Kawamure, and Y. Toba, "Fine structure of laboratory wind-waves surfaces studied using an optical method," Boundary-Layer Meteorol. 39, 133 (1987).

${ }^{3}$ M. Perlin, H. Lin, and C.-L. Ting, "'On parasitic capillary waves generated by steep gravity waves: an experimental investigation with spatial and temporal measurements," J. Fluid Mech. 255, 597 (1993).

${ }^{4}$ M. S. Longuet-Higgins, "The generation of capillary waves by steep gravity waves,' J. Fluid Mech. 16, 138 (1963).

${ }^{5}$ M. S. Longuet-Higgins, "Capillary rollers and bores,' J. Fluid Mech. 240, 569 (1992)

${ }^{6}$ M. S. Longuet-Higgins, "Parasitic capillary waves: a direct calculation," J. Fluid Mech. 301, 79 (1995).

${ }^{7}$ G. D. Crapper, "Non-linear capillary waves generated by steep gravity waves," J. Fluid Mech. 40, 149 (1970).

${ }^{8}$ M. S. Longuet-Higgins, "Progress toward understanding how waves break," in the 21st Symposium on Naval Hydrodynamics (National Academy Press, 1997), pp. 7-28.

${ }^{9}$ B. Chen and P. G. Saffman, "Steady gravity-capillary waves on deep water-I. Weakly nonlinear waves." Stud. Appl. Math. 60, 183 (1979).

${ }^{10}$ B. Chen and P. G. Saffman, "Steady gravity-capillary waves on deep water-II. Numerical results for finite amplitude,'” Stud. Appl. Math. 62, 95 (1980).
${ }^{11}$ L. W. Schwartz and J.-M. Vanden-Broeck, "Numerical solution of the exact equations for capillary-gravity waves,', J. Fluid Mech. 95, 111 (1979)

${ }^{12}$ M. P. Tulin, "Breaking of ocean waves and downshifting," in Waves and Nonlinear Processes in Hydrodynamics (Kluwer Academic, New York, 1996), pp. 177-190.

${ }^{13} \mathrm{P}$. Wang, Y. Yao, and M. Tulin, "An efficient numerical tank for nonlinear water waves, based on the multi-subdomain approach with BEM," Int. J. Numer. Methods Fluids 20, 1315 (1995).

${ }^{14}$ M. S. Longuet-Higgins, "Capillary jumps on deep water," J. Phys. Oceanogr. 26, 1957 (1996).

${ }^{15}$ M. S. Longuet-Higgins and E. D. Cokelet, "The deformation of steep surface waves on water I. A numerical method of computation,'” Proc. R. Soc. London, Ser. A 350, 1 (1976).

${ }^{16} \mathrm{~J}$. W. Dold, "An efficient surface-integral algorithm applied to unsteady gravity waves," J. Comput. Phys. 103, 90 (1992).

${ }^{17}$ D. I. Pullin, "Numerical studies of surface-tension effects in nonlinear Kelvin-Helmholtz and Rayleigh-Taylor instability,' J. Fluid Mech. 119, 507 (1982).

${ }^{18}$ R. Rangel and W. Sirignano, "Nonlinear growth of Kelvin-Helmholtz instability: Effect of surface tension and density ratio,' Phys. Fluids 31, 1845 (1988).

${ }^{19}$ J. T. Beale, T. Y. Hou, and J. S. Lowengrub, "'Convergence of a boundary integral method for water waves,"' SIAM (Soc. Ind. Appl. Math.) J. Numer. Anal. 33, 1797 (1996)

${ }^{20}$ T. Y. Hou, J. S. Lowengrub, and M. J. Shelley, "Removing the stiffness from interfacial flows with surface tension," J. Comput. Phys. 114, 312 (1994).

${ }^{21}$ T. Y. Hou, J. S. Lowengrub, and M. J. Shelley, "The long-time motion of vortex sheets with surface tension," Phys. Fluids 9, 1933 (1997).

${ }^{22}$ H. D. Ceniceros and T. Y. Hou, "Convergence of a non-stiff boundary integral method for interfacial flows with surface tension," Math. Comput. 67, 137 (1998).

${ }^{23}$ G. Baker and A. Nachbin, "Stable methods for vortex sheet motion in presence of surface tension," SIAM J. Sci. Comput. 19, 1737 (1998).

${ }^{24}$ M. Koga, "Bubble entrainment in breaking wind waves,"' Tellus 34, 481 (1982).

${ }^{25}$ T. S. Lundgren and N. N. Mansour, "Oscillations of drops in zero gravity with weak viscous effects,' J. Fluid Mech. 194, 479 (1988).

${ }^{26}$ G. R. Baker, D. I. Meiron, and S. A. Orszag, "Generalized vortex methods for free-surface flow problems," J. Fluid Mech. 123, 477 (1982).

${ }^{27}$ H. D. Ceniceros, "Convergence of a reformulated boundary integral method for two fluid interfaces with surface tension," Ph.D. thesis, New York University, May 1995.

${ }^{28}$ U. M. Ascher, S. J. Ruuth, and B. Wetton, "Implicit-explicit methods for partial differential equations," SIAM (Soc. Ind. Appl. Math.) J. Numer. Anal. 32, 797 (1995).

${ }^{29}$ T. S. Lundgren, "A free surface vortex method with weak viscous effects," in Mathematical Aspects of Vortex Dynamics, edited by R. C. Caflisch (SIAM, Philadelphia, 1989). 